\title{
Bisphosphonate therapy after cardiac transplant reduces fracture risk
}

Fracture risk is reduced and bone mass is preserved in recipients of cardiac transplants treated with the bisphosphonate ibandronate during the first year after transplantation, according to a randomized, placebo-controlled trial conducted by Astrid Fahrleitner-Pammer and colleagues (Medical University of Graz, Austria).

The high doses of immunosuppressive drugs given to transplant recipients in the early post-transplant period cause bone loss and increase risk of fracture. Strategies to prevent these complications have been investigated: bisphosphonate therapy showed promise for prevention of post-transplantation bone loss, but evidence of a beneficial effect on fracture incidence was lacking.

In the current trial, 35 men who had received cardiac transplants were randomly allocated to receive $2 \mathrm{mg}$ ibandronate intravenously every 3 months or a matching placebo; all participants also received calcium and vitamin D supplementation. The first treatment was given within $24 \mathrm{~h}$ of transplantation, as early initiation of therapy seems to be important.

Over the post-transplant year, the mean BMD of patients in the placebo group decreased by $25 \%$ at the lumbar spine and $23 \%$ at the femoral neck; by contrast, mean BMD of ibandronate-treated patients remained the same as at baseline. Analysis of bone-turnover markers revealed that the treatment prevented the marked increase in bone resorption observed in the placebo group. Fracture rate was reduced by ibandronate treatment: incident vertebral fractures occurred in 53\% of the placebo group, but in only $13 \%$ of the ibandronate group. Histomorphometric findings also demonstrated the beneficial effects of ibandronate treatment. Bone biopsy samples from three patients in the placebo group showed a pronounced increase in the percentage of eroded surface between baseline and 6 months, whereas over the same time period, biopsy samples from three patients treated with ibandronate showed a decrease or no change in the percentage of eroded surface.

"The findings of the current analysis underline the importance of bisphosphonate treatment in the early post-transplantation period and may help to establish (parenteral) bisphosphonate therapy as a routine therapy following cardiac transplant," concludes Fahrleitner-Pammer.

\section{Carol Wilson}

Original article Fahrleitner-Pammer, A. et al. Ibandronate prevents bone loss and reduces vertebral fracture risk in male cardiac transplant patients: a randomised double-blind, placebo controlled trial. J. Bone Miner. Res. doi:10.1359/jbmr.090216 